\title{
Cardiac effects of short course dexamethasone in preterm infants
}

\author{
R Skelton, A B Gill, J M Parsons
}

\begin{abstract}
Aim-To examine the incidence and natural history of left ventricular hypertrophy (LVH) associated with the shorter 2-3 week course of dexamethasone, now more usual, for chronic lung disease.

Method-Thirty one infants, gestational age 23-34 (median 26) weeks, birthweight 500-2054 (median 815)g, received dexamethasone, starting at $0.4-0.6 \mathrm{mg} / \mathrm{kg} / \mathrm{day}$, at a median of 11 days of age (range 2-34), weaning over a period of 2-3 weeks. Eighteen preterm neonates were studied as controls over a similar time period. Serial echocardiographic measurements of end diastolic interventricular septum (IVSd) and left ventricular posterior wall (LVPWd) thicknesses were taken before, and up to 48 days after, starting dexamethasone. Maximum Doppler blood flow velocities from the left ventricular outflow tract (LVOT) were measured.

Results-Left ventricular hypertrophy (LVH) occurred in 29 babies (94\%). Median hypertrophy of the IVSd in those receiving dexamethasone was $67 \%$ and LVPWd $56 \%$ of baseline measurements, significantly greater than control infants $(p<0.001)$. LVH appeared by a median of three days, peaking by a median of 10 days. All resolved by a median of 27 days. LVOT obstruction was not seen. There was no significant correlation with birthweight, gestation, blood pressure, or glucose tolerance.

Conclusions-LVH developed in almost all preterm neonates receiving a $2-3$ week course of dexamethasone, but was of little clinical importance and always resolved. Echocardiography is probably not required routinely in infants receiving such short course dexamethasone for chronic lung disease.
\end{abstract}

(Arch Dis Child Fetal Neonatal Ed 1998;78:F133-F137)

Keywords: dexamethasone; chronic lung disease; left ventricular hypertrophy; echocardiography

Dexamethasone is a very effective treatment for weaning preterm infants with clinically significant lung disease from the ventilator. ${ }^{12}$ However, it is not without side effects. Early reports of 6 week regimens suggest clinically significant cardiac effects, particularly thickening of the wall of the left ventricle-left ventricular hypertrophy $(\mathrm{LVH})^{3}{ }^{4}$ - which can lead to symptomatic left ventricular outflow tract obstruction (LVOT). This led to the recommendation that infants receiving dexam- ethasone should undergo regular echocardiography. ${ }^{4}$ Despite extensive use of dexamethasone in neonatal practice over the past 10 years, little is known about the natural history of the LVH.

Dexamethasone was originally introduced as a 6 week course, from 28 days of age, to treat established chronic lung disease (CLD). ${ }^{1}$ Courses are now becoming shorter and are often started earlier, in an attempt to reduce lung injury and prevent CLD. ${ }^{2}$ Most units now use similar doses to those used in the original trials, but start by the second week of life, weaning over 2-3 weeks rather than $6 .{ }^{2}$ Despite the apparent clinical effectiveness of early, short course dexamethasone, little is known about its side effects. To our knowledge, the effects on the heart of this shorter, earlier course of dexamethasone have not yet been studied.

Our aims were to establish whether LVH occurs with an early, 3 week course of dexamethasone in preterm neonates, in a prospective, longitudinal study, and to investigate its incidence, natural history, severity and clinical importance. We also re-examined recommendations for routine echocardiography.

\section{Methods}

The study received local ethics committee approval. All preterm babies ( $<36$ weeks of gestation), admitted to two regional neonatal intensive care units in Leeds (at Leeds General Infirmary and St James's University Hospital), receiving dexamethasone for lung disease between May and November 1995, were studied. As little is known about the changes in left ventricular morphology in extremely preterm infants, we also collected data from similarly aged infants who did not receive dexamethasone and who acted as controls. Babies with congenital heart disease and diabetic mothers were excluded from the control population.

Basic clinical variables were recorded, including measurements of blood pressure, blood and urine glucose measurements for the previous 12 hours, carbohydrate intake, insulin requirements and ventilation and oxygen requirements. Glucose intolerance was defined as urine glucose of $1+$ or greater, $\mathrm{BM}$ stix $>10$ $\mathrm{mmol} / \mathrm{l}$, the need to reduce carbohydrate intake, or use insulin. Dexamethasone was given at a dose of $0.4-0.6 \mathrm{mg} / \mathrm{kg} / \mathrm{day}$, weaning over 15-23 days in 5-7 day reductions.

A Toshiba 140A scanner was used at Leeds General Infirmary and a Hewlett Packard Sonos 100 scanner at St James Hospital. A 7.5 MHZ probe was used in all cases. The examinations were done by one person (RS). Firstly, 
Table 1 Demographic details for dexamethasone treated infants and controls (all values are median (range))

\begin{tabular}{lcc}
\hline & Dexamethasone group $(n=31)$ & Controls $(n=18)$ \\
\hline Gestation (weeks) & $26(23-34)$ & $29^{\star}(25-34)$ \\
Birthweight (g) & $815(500-2045)$ & $1215^{\star}(760-1530)$ \\
Age treatment started (days) & $11(2-34)$ & \\
M:F ratio & $23: 8$ & $8: 10$ \\
Centiles & $39(1-80)$ & $33(5-90)$ \\
\% inspired oxygen & $37(22-100)$ & $30(21-70)$ \\
Insulin required & 4 & 0 \\
\hline
\end{tabular}

^Mann Whitney, $\mathrm{p}<0.01$.

the left ventricle was studied from cross sectional, parasternal long axis views. Measurements of end diastolic interventricular septal (IVSd) and left ventricular posterior wall (LVPWd) thickness, and left ventricular internal diameter (LIDDd) were taken on M-mode at the level of the mitral valve leaflets. ${ }^{5}$ Measurements were averaged for three to five cardiac cycles. The presence of a patent ductus arteriosus (PDA) was noted by direct imaging of the duct and Doppler studies. Serial short axis views of the left ventricle (LV) were taken from the level of the mitral valve leaflets to the apex, to assess the distribution of any LVHwhether it affected the ventricle globally, segmentally, or apically. The left ventricular outflow tract (LVOT) was visualised on apical 5 chamber views and, where possible, the maximum velocity (Vmax) was measured using continuous or pulsed wave Doppler.

Infants who received dexamethasone had a baseline echocardiograph before the administration of dexamethasone, usually within 24 hours. Echocardiographs were then repeated two to three times a week, for two weeks and weekly thereafter until measurements either reached baseline or steady state. Control babies were examined on days 7 and 28 of life. Intraobserver error was calculated using the method of Bland and Altman ${ }^{6}$ for two factors: the variability between scans and the variability between measurements of a single scan. Seven babies were re-scanned immediately after the initial scan and 18 scans were re-measured. This produced good coefficiency of repeatability of $0.4-0.5 \mathrm{~mm}$ for measurements of IVSd and LVPWd and $0.9-1 \mathrm{~mm}$ for measurements of LIDDd.

LVH associated with dexamethasone was defined as an increase in the thickness of the wall of the left ventricle (an increase in IVSd or LVPWd thickness) significantly greater than that of the control infants (greater than 2 standard deviations from the mean), with a concomitant reduction in the LIDDd. For those in whom LVH occurred, the following were then analysed: the incidence of $\mathrm{LVH}$; its

Table 2 Severity of LVH in 24 infants receiving dexamethasone and 18 controls (median and ranges)

\begin{tabular}{|c|c|c|c|c|}
\hline & $\begin{array}{l}\text { Increase in IVSd } \\
\text { mmts (mm) }\end{array}$ & $\begin{array}{l}\text { IVSd (\% of } \\
\text { baseline) }\end{array}$ & $\begin{array}{l}\text { Increase in LVPWd } \\
m m t(\mathrm{~mm})\end{array}$ & $\begin{array}{l}\text { LVPWd } \\
\text { (\% baseline) }\end{array}$ \\
\hline \multicolumn{5}{|c|}{ Dexamethasone } \\
\hline Median & $1.6^{\star}$ & $67 \%$ * & $1.3^{\star}$ & $56 \%$ * \\
\hline Range & $(1.1-3.1)$ & $(26-176)$ & $(0.5-2.5)$ & $(26-200)$ \\
\hline \multicolumn{5}{|l|}{ Controls } \\
\hline Median & 0.1 & $3 \%$ & 0.3 & $15 \%$ \\
\hline Range & $(-0.8-1)$ & $(-24-37)$ & $(-0.7-1.6)$ & $(-19-94)$ \\
\hline
\end{tabular}

^ Mann Whitney $\mathrm{p}<0.001$ compared with controls. distribution-whether it affected the ventricle globally, segmentally, or apically; and symmetry in terms of relative thickness of the IVSd and LVPWd - severity, timing, and ductal patency. The severity of the LVH was calculated as the maximum increase in thickness of both the IVSd and LVPWd from baseline measurements. This was given both as actual measurements and percentage of baseline measurements. Median and interquartile ranges were calculated. Measurements of infants receiving dexamethasone were not normally distributed, so Mann Whitney $U$ tests were used to compare the increase in IVSd and LVPWd thicknesses between both groups of infants, and between baseline and subsequent measurements in each group.

Spearman rank correlation and Mann Whitney tests were carried out on the following variables to assess for correlation with timing and severity of LVH: the development of glucose intolerance; insulin use; day of life steroids were started; sex; gestation; birthweight and centile. For blood pressure, a correlation was sought between blood pressure values and LVH and a Mann Whitney test comparing the change in left ventricular thickness in infants with an arbitrary increase of over $10 \mathrm{~mm} \mathrm{Hg}$ after starting dexamethasone. Significance was taken as $\mathrm{p}<0.05$. ASTUTE on Microsoft EXCEL was used for all statistical calculations.

\section{Results}

Thirty one babies received dexamethasone and were examined over six months. Dexamethasone was started at a median of 11 days of age with a range of 2 to 34 days. The baseline scans were done at a median of 0 days before starting dexamethasone (the same day as treatment), range 0-9 days; 18 control babies were examined at 7 and 28 days of age. Those who received dexamethasone were significantly smaller and more premature than the controls (table 1).

A slight increase was seen in the thickness of both the IVSd and LVPWd (table 2) and in the internal diameter of the left ventricle over the 21 days among the control infants. This was not significant compared with baseline measurements $(p=0.33)$. Twenty nine of the 31 neonates who received dexamethasone (94\%) developed $\mathrm{LVH}$, according to our definition. The pattern of the LVH over time is shown in figs 1 and 2. It was always concentric, with no preferential thickening of either the interventricular septum or the posterior wall.

Of these 29 infants, three died, two were transferred out by day 7 , leaving 24 in whom maximal hypertrophy could be assessed. A further nine were transferred later, leaving 15 still available for assessment through to full resolution (table 3). None died directly as a result of dexamethasone or LVH. There was no difference between the infants transferred and those who remained.

The maximum degree of LVH in the 24 infants receiving dexamethasone is shown in table 2. The LIDDd decreased in parallel with the $\mathrm{LVH}$, with a median decrease of $43 \%$ (range $0-60 \%$ ). IVSd and LVPWd thickness 


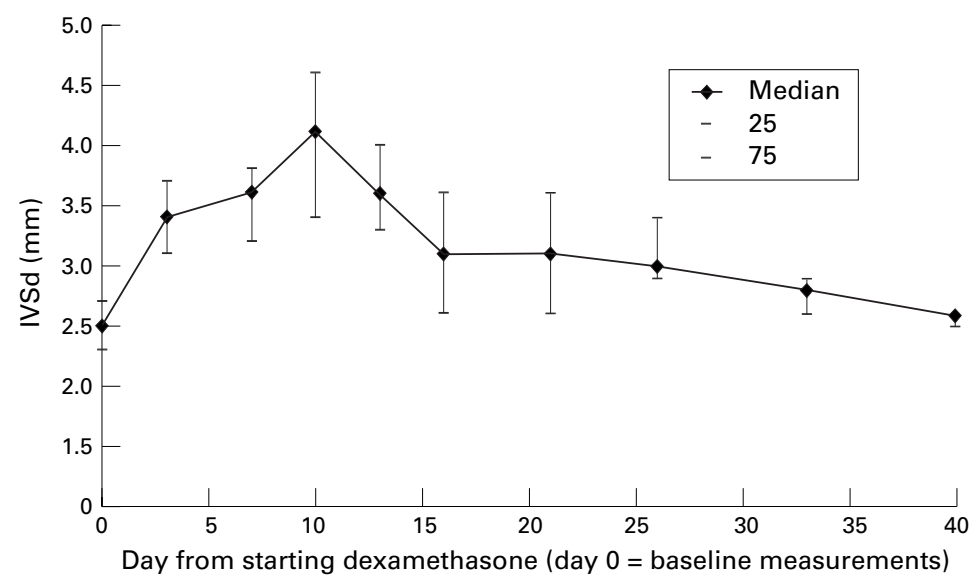

Figure 1 Measurements of IVSd thickness in infants receiving dexamethasone from the start of treatment up to 40 days (median and interquartile range).

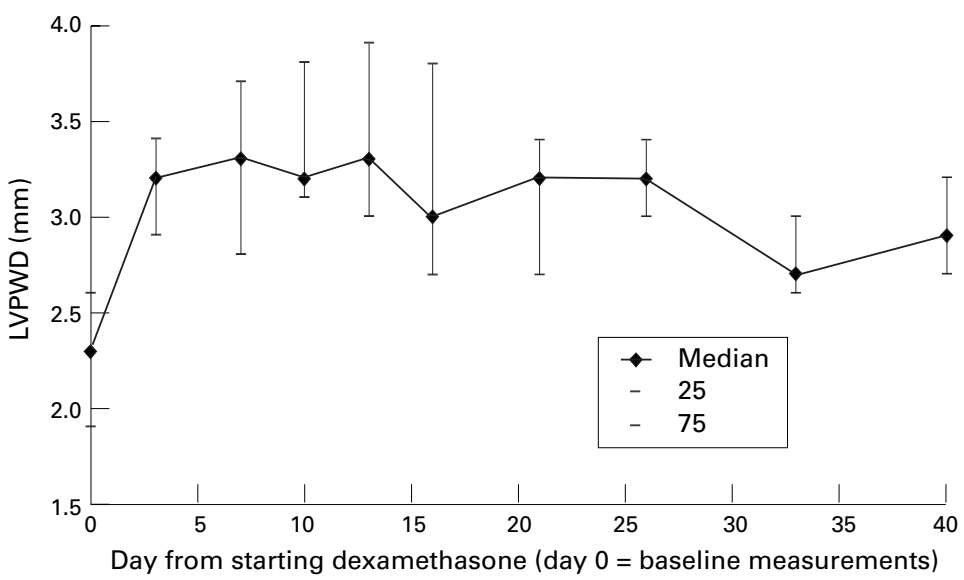

Figure 2 Measurements of LVPWd thickness of infants receiving dexamethasone from the start of treatment up to 40 days (median and interquartile range).

were significantly greater than baseline measurements on each examination up to 22 days after starting dexamethasone $(p<0.05)$. The maximum increase in IVSd and LVPWd in the group of infants receiving dexamethasone was significantly greater than the increase seen in the control infants between days 7 and 28 of age (table 2).

Three neonates developed very severe LVH of over $100 \%$ of baseline. They were all very premature (23-28 weeks), growth retarded (under 3rd centile), and two received insulin.

Timing of development and resolution of the LVH are shown in table 3. LVH developed very quickly, usually within two to three days. Maximum hypertrophy occurred by about 2 weeks, with resolution often beginning before the dexamethasone was fully weaned. Fifteen surviving infants were followed up throughout the regional centres. LVH resolved in all of these infants.

Peak Doppler blood flow velocities (Vmax) were recorded in 14 neonates in the LVOT

Table 3 Timing of onset, maximum and resolution of LVH associated with short course of dexamethasone

\begin{tabular}{llll}
\hline & Median day & Range & Number \\
\hline Onset & 3 & $2-6$ & 29 \\
Maximum & 10 & $2-25$ & 24 \\
Resolution & 26 & $14-39$ & 15 \\
\hline
\end{tabular}

during dexamethasone treatment, with a median of $0.76 \mathrm{~m} / \mathrm{s}$, range $0.42-1.75 \mathrm{~m} / \mathrm{s}$. Twelve babies had a PDA, with a left to right shunt, at the onset of treatment with dexamethasone. This remained patent in three by the end of treatment and in two at death at 3 days of age. In six the PDA closed at a range of three to 12 days of treatment. Surgical ligation was required in one baby during treatment with dexamethasone.

There were few side effects. Intestinal perforation was seen in two infants. None showed any obvious clinical effects of the $\mathrm{LVH}$, although one developed a murmur on day 14, at the time of her maximum LVH. The Vmax in the LVOT was $1.75 \mathrm{~m} / \mathrm{s}$, with turbulent flow, but she remained asymptomatic throughout.

Gestational age, birthweight, and the age at which dexamethasone was started showed no significant correlation with LVH. Twelve babies had glucose intolerance that was present before dexamethasone in five. Four babies required insulin, two as a continuous infusion (both started before dexamethasone) and two as a single bolus. There was no correlation between timing or severity of LVH and glucose tolerance. Blood pressure showed no correlation by either criteria used. No control baby required insulin.

\section{Discussion}

The original studies on the use of dexamethasone clearly showed a beneficial effect in weaning infants from the ventilator. ${ }^{2}$ The recommendations were for a six week course, from 28 days of age, for infants with established CLD. However, earlier courses are now advocated in an attempt to limit the development of severe CLD, often seen with more delayed treatment. Additional concerns regarding adverse effects on growth and development ${ }^{2} 7$ have resulted in many units changing to a regimen of dexamethasone at around the second week of life, tapering over only two to three weeks. This has been shown to be comparable in effectiveness with a six week course. ${ }^{2}$ All previous reports of cardiac effects of dexamethasone were on infants on the original six week or longer courses from 4 weeks of age. Our study is more applicable to the present day practice of shorter, earlier courses.

There are difficulties in tightly defining $\mathrm{LVH}$ in a group in whom there are few standard criteria. We have clearly shown a large, transient, increase in the thickness of the left ventricular wall, affecting both the IVSd and the LVPWd, that is associated with a decrease in the LIDDd in infants receiving dexamethasone. This increase was very obvious on real time scanning. It contrasts with the control infants in whom only a very slight increase in the IVSd, LVPWd was seen, associated with a increase in the LIDDd. This increase was not significant and the real time picture seen was very different. Thus the increase in IVSd and LVPWd thickness in infants receiving dexamethasone seems to be true hypertrophy (LVH) rather than normal postnatal growth.

LVH occurred in $94 \%$ of infants treated with dexamethasone. The degree of LVH was very 
similar to that seen by Werner ${ }^{3}$ and Evans ${ }^{4}$ in infants receiving a six week course, although Bensky ${ }^{8}$ found a smaller effect with an increase of only $15-20 \%$. The distribution is different from that seen in other conditions. In familial hypertrophic cardiomyopathy (HCM) and infants of mothers with diabetes, the hypertrophy usually affects the septum, rather than the left ventricular posterior wall ${ }^{10}$ and in HCM the ventricle may also be affected in isolated segments, especially apical. Our results agree with those of Israel ${ }^{11}$ and Bensky ${ }^{8}$ that the LVH associated with dexamethasone seems to be more uniform, affecting the septum and free wall to an equal extent.

The appearances of the heart change quite dramatically and quickly after starting dexamethasone. Maximal LVH usually developed by the end of the second week, with resolution often beginning during weaning of the dexamethasone. About half of our infants either died or were transferred back to their referring hospital before full resolution could be determined. The LVH in the infants who died was comparable with that in the rest of the group and there is no reason to suspect that the resolution of LVH of those transferred back to base hospitals would be any different from those infants in whom full follow up was possible. Our finding supports those of Israel ${ }^{11}$ in that shortening the treatment produces more rapid reversal of changes.

The first description of LVH associated with steroids in preterm neonates appeared in 1990. ${ }^{12}$ Four babies developed severe LVH after a six week course of dexamethasone. Three developed clinically significant left ventricular outflow tract obstruction. Since then a further eight reports have recorded $\mathrm{LVH}$, in a total of 57 babies, receiving $0.5-1$ $\mathrm{mg} / \mathrm{kg}$ day of dexamethasone, reducing over six weeks or longer. ${ }^{34811-15}$ These reports have either only reported infants with LVOTO, ${ }^{12-15}$ or have quoted an incidence of up to $18 \%$ of those studied, ${ }^{48}$ often referring to infants with turbulent flow in the LVOT, or maximum blood flow velocities of more than $2 \mathrm{~m} / \mathrm{s}$. Despite quite considerable LVH, we saw no real left ventricular outflow tract obstruction (LVOTO) with our shorter course. Although the normal ranges of maximum blood flow velocities are not known for this group of preterm infants, they were always within usually accepted normal ranges. Despite a similar starting dose, shorter courses of dexamethasone do not seem to produce the same effect on the outflow tract, and the severity of LVH seems to be related to the duration of treatment. Lower doses have not been studied, so we do not know whether a lower starting dose would cause even less LVH, with similar clinical benefits.

There was no significant correlation between LVH and gestation, birthweight, age at which dexamethasone was started, blood pressure, hyperglycaemia or insulin use. However, three infants developed a particularly large increase in the thickness of their IVSd and LVPWd, of over $100 \%$ of their baseline measurements. These were very small infants - two received insulin-suggesting a link between insulin treatment and dexamethasone associated LVH. Small numbers may explain the lack of a significant difference between those who received insulin with dexamethasone and those who received dexamethasone alone. It is still possible that a small high risk group of preterm infants exists for $\mathrm{LVH}$ with dexamethasone. Starting the dexamethasone did not have any obvious effect on the PDA.

What, then, is the possible mechanism of the LVH associated with dexamethasone treatment? It does not seem to be directly related to systemic or pulmonary hypertension. ${ }^{4}$ In preterm infants LVH has also been described in association with CLD. ${ }^{16}$ The mechanism is thought to be secondary to raised pulmonary artery pressure that is attendant on unrecognised hypoxia. ${ }^{17}$ However, the LVH seen in infants receiving dexamethasone appears much more quickly than with CLD or hypoxia and resolves very rapidly on weaning the dexamethasone.

LVH is also seen in conditions with abnormal glucose or insulin metabolism. ${ }^{18-20}$ The natural history of LVH associated with dexamethasone suggests a direct effect on the heart through these pathways. At a cellular level, cardiac hypertrophy usually results from hypertrophy of the myocytes, rather than division, by the synthesis of various intracellular cardiac proteins, rather than deposition of substances within the cell. ${ }^{21}$ Synthesis of such cardiac proteins can occur within 48 hours of an appropriate stimulus on cardiac receptors. This timing correlates well with the speed of LVH seen after starting dexamethasone treatment. Stimuli known to lead to myocyte hypertrophy in vivo and vitro include stretch, hypertension, adrenaline, endothelin, insulin and IGF1. ${ }^{21}$ IGF1 can directly stimulate skeletal muscle growth. ${ }^{22}$ In adult myocytes stretch and increased work is usually thought to be the stimulus for LVH. However, in neonates stimulation of cardiac insulin and IGF1 receptors seem to be more important. The density of IGF1 and insulin receptors are at their highest in neonates and preterm infants and decrease during infancy. ${ }^{22-24}$ An effect of dexamethasone through IGF1 or its receptors directly on cardiac myocytes would explain entirely the dexamethasone induced LVH. Insulin will act on the heart in addition to the dexamethasone, and this may explain the greater degree of hypertrophy in those infants receiving insulin.

In conclusion a short two to three week course of dexamethasone produces a transient LVH in almost all infants. The clinical effect seems to be less severe than with a six week course and as such we would not recommend routine echocardiography of all infants receiving dexamethasone. LVH associated with short courses of dexamethasone seemed to be relatively benign, but caution should still be exercised, particularly in very small neonates receiving insulin, as no long term data are available. Further studies are required more fully to understand the cause and variability of this LVH and its risk factors and long term prognosis. These could be combined with fur- 
ther work to ascertain the most effective and lowest dose of dexamethasone for infants with CLD.

We thank Drs P Dear and S Newell and all the staff at St James University Hospital, Leeds for allowing us to examine their babies.

1 Ehrenkranz RA, Mercurio R. Bronchopulmonary dysplasia. In: Sinclair J, Bracken MB, eds. Effective care of the newborn infant. Oxford: Oxford University Press, 1992:399-424.

$2 \mathrm{Ng}$ PC. The effectiveness and side effects of dexamethasone in preterm infants with bron

3 Werner JC, Sicard RE, Hansen TWR, Solomon E, Cowett RM, Oh W. Hypertrophied cardiomyopathy associated with dexamethasone therapy for bronchopulmonary dysplasia. F Pediatr 1992;120:286-91.

4 Elasia. F Pediatr 1992;120:286-91. preterm infant. Arch Dis Child 1994;70:F25-F30.

5 Henry WL, DeMaria A, Gramiak R, King DL, Kisslo JA Popp RL, et al. Report of the American Society of Echocardiography standards in 2 dimensional echocardiography. Circulation $1980 ; 62: 212-7$

6 Bland JM, Altman DG. Statistical methods for assessing agreement between two methods of clinical measurement. Lancet 1986;ii:307-10.

7 Brownlee KG, Ng PC, Henderson MJ, Smith M, Green JH, Dear PRF. Catabolic effect of dexamethasone in the preterm baby. Arch Dis Child 1992;67:1-4.

8 Bensky AS, Kothadia JM, Covitz W. Cardiac effects of dexamethasone in very low birthweight infants. Pediatrics 1996;96:818-21.

9 Feigenbaum H. Cardiomyopathies. In: Feigenbaum H, ed Echocardiography. 5th edn. Pennsylvania: Lea and Febiger, Echocardiogra

$10 \mathrm{Katz}$ VL, Bowes WA. Maternal diseases affecting the fetal cardiovascular system. In: Long W, ed. Fetal and Neonatal cardiovascular system. In: Long W, ed. Fetal and
Cardiology. Toronto: WB Saunders, 1990:137-8.

11 Israel BA, Sherman FS, Guthrie RD. Hypertrophied cardiomyopathy associated with dexamethasone therapy for chronic lung disease in preterm infants. Am F Perinato 1993:10:307-10

12 Shuster D, Gidding SS. Possible relationship of acquired hypertrophic cardiomyopathy to corticosteroid therapy in premature infants with bronchopulmonary dysplasia. Pediatr Res 1990;27:237A.

13 Brand PLP, van Lingen RA, Brus F, Talsma MD, Elzenga NJ. Hypertrophic obstructive cardiomyopathy as a side effect of dexamethasone treatment for bronchopulmonary dysplasia. Acta Paediatr 1993;82:614-7.

14 Ohning BL, Fyfe DA, Riedel PA. Reversible obstructive hypertrophic cardiomyopathy after dexamethasone therapy for bronchopulmonary dysplasia. Am Heart $\mathcal{F}$ 1993;123:253-6.

15 Haney I, Lachance C, Fouron JC, Van Doesburg NH. Reversible steroid induced hypertrophic cardiomyopathy with left ventricular outflow tract obstruction. $A m \mathcal{F}$ Perinatol 1995;12:271- 4.

16 Melnick G, Pickoff AS, Ferrer PL, Peyser J, Bancalari E, Gelband $H$. Normal pulmonary vascular resistance and LVH in young infants with bronchopulmonary dysplasia: An echocardiographic and pathological study. Pediatrics An echocardiograpt

17 Gill $\mathrm{AB}$, Weindling $\mathrm{AM}$. Raised pulmonary artery pressure in very low birthweight infants requiring supplemental oxygen at 36 weeks after conception. Arch Dis Child $1995 ; 72 ; 20-2$

18 Breitweser JA, Meyer RA, Sperling MA, Tsang RC, Kaplan S. Cardiac septal hypertrophy in hyperinsulinaemic infants. F Pediatr 1980;96:535-9.

19 Fox LA, Geffner ME, Al Khatib Y, Kaplan S. Hyperinsulinaemic, hypertrophic cardiomyopathy in infancy. Am $\mathcal{F} D$ is Child 1992; 146:896-9.

20 Carter BS, DiGiacomo JE, Balderston SM, Wiggins JW, Merenstein GB. Disproportionate septal hypertrophy associated with erythroblastosis fetalis $\mathrm{Am} \mathrm{f}$ Dis Child 1990;144:1225-8.

21 Glennon PE, Sugden PH, Poole-Wilson PA. Cellular mechanisms of cardiac hypertrophy. $\mathrm{Br}$ Heart $\mathcal{f}$ 1995; 73:496-9.

22 Rosenfeld RG, Gargosky SE. Assays for insulin-like growth factors and their binding proteins: practicalities and pitfalls. F Pediatr 1996;128:S52-7.

23 Rajaram S, Carlson SE, Koo WWK, Rangachari A, Kelly DP. Insulin-like growth factor (IGF)-1 and IGF-binding protein 3 during the first year in term and preterm infants. protein 3 during the first year
Pediatr Res $1995 ; 37: 581-5$.

24 Thorsson AV, Hintz RL. Insulin receptors in the newborn. Increase in receptor affinity and number. $N$ Engl $\mathcal{F} \mathrm{Med}$ 1977;297:908-12. 